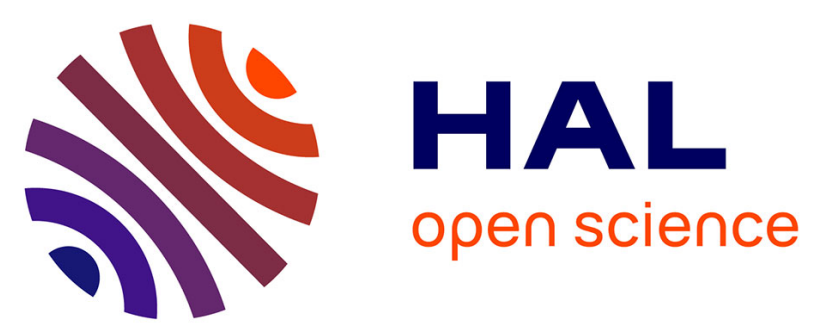

\title{
Cooperative oscillation of non-degenerate transverse modes in an optical system: multimode operation in parametric oscillators
}

Axelle Amon, Pierre Suret, Serge Bielawski, Dominique Derozier, Marc Lefranc

\section{To cite this version:}

Axelle Amon, Pierre Suret, Serge Bielawski, Dominique Derozier, Marc Lefranc. Cooperative oscillation of non-degenerate transverse modes in an optical system: multimode operation in parametric oscillators. Physical Review Letters, 2009, 102, pp.183901. 10.1103/PhysRevLett.102.183901 . hal$00288468 \mathrm{v} 3$

\section{HAL Id: hal-00288468 \\ https://hal.science/hal-00288468v3}

Submitted on 11 May 2009

HAL is a multi-disciplinary open access archive for the deposit and dissemination of scientific research documents, whether they are published or not. The documents may come from teaching and research institutions in France or abroad, or from public or private research centers.
L'archive ouverte pluridisciplinaire HAL, est destinée au dépôt et à la diffusion de documents scientifiques de niveau recherche, publiés ou non, émanant des établissements d'enseignement et de recherche français ou étrangers, des laboratoires publics ou privés. 


\title{
Cooperative oscillation of non-degenerate transverse modes in an optical system: multimode operation in parametric oscillators
}

\author{
Axelle Amon, ${ }^{1,2}$ Pierre Suret, ${ }^{3,4}$ Serge Bielawski, ${ }^{3,4}$ Dominique Derozier, ${ }^{3,4}$ and Marc Lefranc ${ }^{3,4}$ \\ ${ }^{1}$ Université de Rennes 1, Institut de Physique de Rennes, Campus de Beaulieu, F-35042 Rennes, France. \\ ${ }^{2}$ CNRS, UMR 6251, F-35042 Rennes, France. \\ ${ }^{3}$ Université des Sciences et Technologies de Lille, Laboratoire de Physique des Lasers, \\ Atomes, Molécules, CERLA, F-59655 Villeneuve d'Ascq, France. \\ ${ }^{4}$ CNRS, UMR 8523, FR 2416, F-59655 Villeneuve d'Ascq, France.
}

(Dated: May 11, 2009)

\begin{abstract}
We show experimentally that parametric interaction can induce a cooperative oscillation of non simultaneously resonant transverse modes in an optical parametric oscillator. More generally, this effect is expected to occur in any spatially extended system subjected to boundary conditions where nonlinear wave mixing of two nonresonant spatial modes can generate a resonant oscillation.
\end{abstract}

PACS numbers: 42.65.-k, 42.65.Sf, 42.65.Yj

Sources of coherent radiation such as lasers or optical parametric oscillators (OPOs) are based on an optical cavity recycling the generated radiation into the gain medium. The cavity imposes longitudinal and transverse boundary conditions on the radiation field and forces it into a superposition of modes, each associated with an oscillation frequency [1, 2]. An important question is whether an optical oscillator can operate simultaneously on several modes. When mode competition for gain is weakened by effects such as spatial, spectral or polariza-

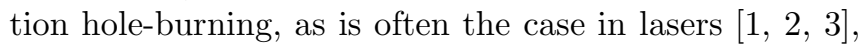
intermode coupling is reduced and multimode operation is common. When instead modes compete strongly, the lowest-threshold mode prevents the onset of other modes (as, e.g., in some homogeneously broadened lasers [1, 2] and optical parametric oscillators [4, (5]), and single-mode operation is the rule ("winner-takes-all" dynamics). This mode selection problem is in fact not specific to optics and is more generally relevant to all spatially extended systems where boundary conditions single out a set of nearly resonant modes which receive energy from a common source and interact nonlinearly [6, 7].

In the "winner-takes-all" case, it is generally believed that multimode operation can only be observed when several modes have identical resonance frequencies and thresholds [5, 8, 9, 10]. In optics, such coincidences occur in degenerate cavities where different combinations of longitudinal and transverse mode indices result in the same oscillation frequency. Except in highly degenerate cavities such as the plane-parallel, confocal and concentric two-mirror configurations [1], mode coincidence is difficult to achieve and thus multimode operation is generally considered to be accidental and uncommon.

However, this view assumes a kind of superposition principle, namely that modes must oscillate individually before they can interact (see, e.g., [6]). In this Letter, we show that even in a "winner-takes-all" system, the nonlinearity of the gain medium can induce simultaneous oscillation of transverse modes with well separated resonance frequencies. More precisely, we experimentally study multimode operation in a continuous-wave triply resonant optical parametric oscillator (TROPO), where optical amplification relies on three-wave mixing [4. Contrary to the common view that the TROPO is a single-mode device [4, 同, except in degenerate configurations [8, 9, 10], we provide experimental evidence of mode coexistence between two monomode resonances. Moreover, we observe multimode operation in many more geometries than predicted from mode coincidences, in particular far from transverse degeneracy. Our findings are consistent with predictions of Ref. [11] and are similar to recent observations in Faraday experiments in hydrodynamics [7]. This suggests that the effect we describe here in an optical system may more generally occur in systems which combine a strong nonlinear interaction with tight boundary conditions.

In spite of its simplicity, the TROPO has a rich dynamics, still only partially understood. It has been predicted theoretically that the monomode TROPO displays a Hopf bifurcation and a period-doubling cascade leading to chaos [12]. Experimentally, fast periodic instabilities have been observed in TROPOs at frequencies in the range 1-300 MHz [13, 14]. They occur alone or combined with slow thermo-optic instabilities [15, 16], giving then rise to bursting oscillations [11], and can follow a transition to chaos [17]. However, fast oscillations can be observed at weaker pumps, smaller signal frequency detunings and with smaller frequencies than predicted for the monomode instability [11, 14, 18].

Using a degenerate two-mode TROPO model [8], it was conjectured in 14] that these oscillations result from the interaction of transverse modes, and shown in [11] that two transverse modes with disjoint resonance curves can interact when their resonance frequencies approximately add up to the pump frequency, generating oscillations at frequencies much higher than cavity bandwith. Conversion of a pump photon into two photons in the same mode is then strongly repressed, leaving the cross-conversion 
channel (one photon in each of the two modes) as the dominant process. Roughly speaking, the OPO views the two combined signal-idler modes as a pair of distinct signal and idler fields, and can thus utilize the fact that parametric interaction only constraints the sum of the generated frequencies, not their individual values.

An important result of Ref. [11] is that an exact expression for the two-mode threshold can be computed, and that it is identical to that of a virtual single-mode regime with an effective coupling constant that depends on mode overlap. This allows one to study the mode selection process in a unified way by considering simultaneously single-mode and two-mode resonances, in which the two neighboring modes oscillate cooperatively (Fig. 1). Because multimode operation is thus allowed to occur without transverse mode degeneracy, it is expected that such regimes can be observed in a large number of cavity configurations.

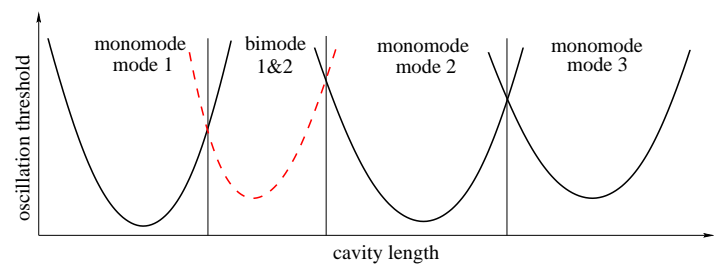

FIG. 1: Schematic representation of the resonance spectrum showing oscillation threshold of various regimes as a function of cavity length. Between two single-mode resonances, there exist two-mode resonances where the two modes of the neighboring resonances oscillate cooperatively.

The experimental setup is similar to that used in previous experiments [11, 14, 15, 16, 17. A 15-mm-long KTP crystal cut for type-II phase matching is enclosed inside a cavity of length $L$ varying between 30 and 100 $\mathrm{mm}$, delimited by two mirrors with a radius of curvature of $50 \mathrm{~mm}$. Cavity length is not actively stabilized. Cavity finesses at $532 \mathrm{~nm}$, the wavelength of the frequencydoubled Nd:YAG pump laser, and at $1064 \mathrm{~nm}$, near which the signal and idler fields are generated, are 50 and 550, respectively. The regimes discussed here have been obtained at a pump power of $3.5 \mathrm{~W}$, with a parametric threshold ranging from $10 \mathrm{~mW}$ to $35 \mathrm{~mW}$ depending on cavity length. The output pump beam had a $\mathrm{TEM}_{00}$ structure. In most configurations, scanning the cavity through one free spectral range with a PZT allowed us to observe fast oscillations in the output intensity of the OPO at some cavity lengths, at a frequency $f$ varying typically from 1 to $300 \mathrm{MHz}$, combined or not with optothermal oscillations (Fig. 2).

During fast oscillations, the transverse profiles of output signal beam observed with a CCD camera suggest the presence of several transverse modes (Fig. 3(a) and (b)). However, the pattern displayed by the camera is averaged over $20 \mathrm{~ms}$, which does not allow us to distin-
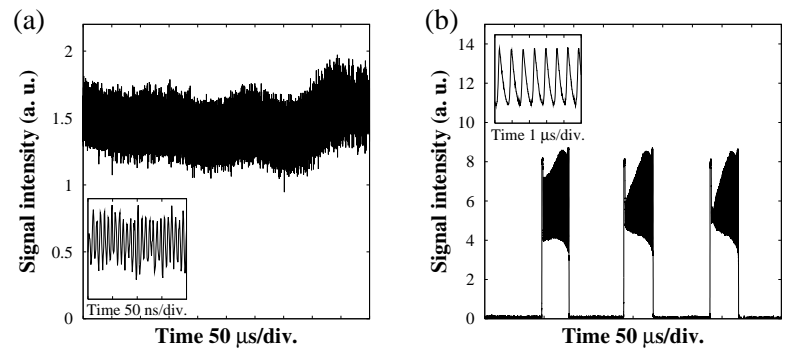

FIG. 2: Signal intensity vs. time in two configurations. (a) $L=47 \mathrm{~mm}$; threshold $10 \mathrm{~mW}, f=130 \mathrm{MHz}$; (b) $L=77$ $\mathrm{mm}$; threshold $33 \mathrm{~mW}, f=1.2 \mathrm{MHz}-2.7 \mathrm{MHz}$.

guish between true mode coexistence and fast periodic alternation [16]. Fig. 3(c) shows a mode analysis using a scanning Pérot-Fabry interferometer (PF) of the output signal in a putative multimode regime. It displays two peaks separated by approximately $1.1 \mathrm{GHz}$, which is much larger than the cavity bandwith. No mode hopping can be seen on the output signal during the scan so that this again suggests coexistence of well-separated modes, but is not conclusive because the two modes are resolved at different times of the scan.

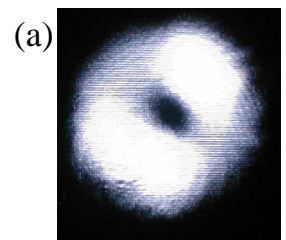

(b)
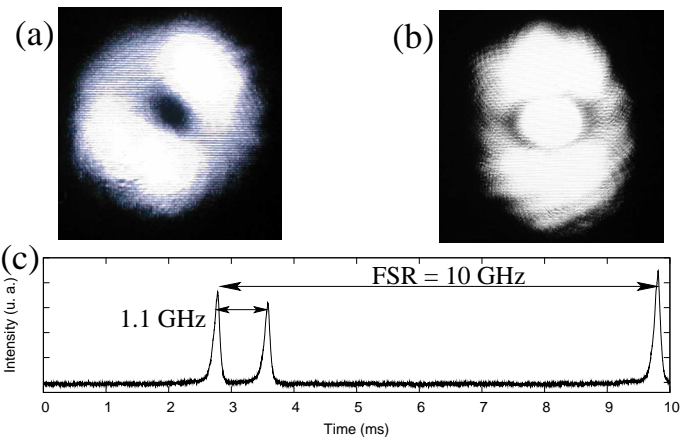

FIG. 3: Transverse intensity profile of the signal field recorded with a standard CCD camera operating at 50 frames/s. (a) $L=46.3 \mathrm{~mm}, f=15 \mathrm{MHz}$; (b) $L=37 \mathrm{~mm}, f=31.5$ MHz. (c) Intensity of the OPO signal at the output of a $\mathrm{PF}$ analyser which cavity length is swept with time. The transmission presents two maxima over one FSR of the PF cavity $(10 \mathrm{GHz})$. The two modes are separated by at least $1.1 \mathrm{GHz}$ (for OPO cavity: $L=47 \mathrm{~mm}$, FSR: $2.5 \mathrm{GHz}$ ).

To obtain convincing evidence of mode coexistence, we have therefore monitored the output intensity of the signal field in two different locations of the transverse plane using fast detectors, although this method probably prevents us to detect very large beat frequencies. A monomode transverse profile is described by a single time-dependent amplitude: the time traces of the two detectors should then be proportional to each other. Fig. A shows the two time traces in a regime where bursts of fast oscillations are observed. The two traces have very different waveforms and are out of phase during the fast oscillations, which shows the presence of at least two modes. 
This clearly confirms that fast oscillations originate in the interaction of two or more transverse modes.
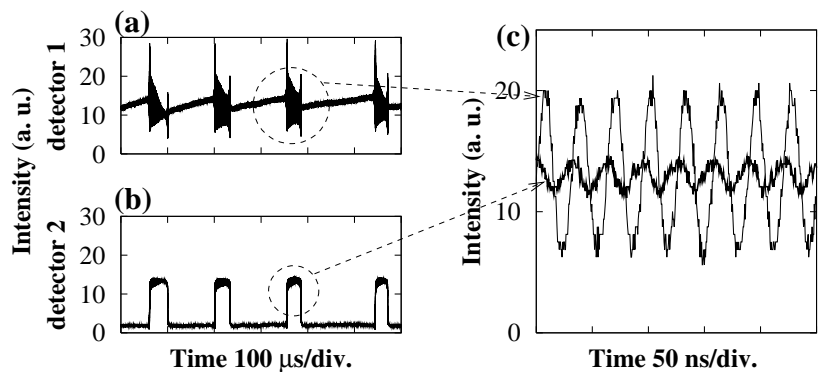

FIG. 4: Time traces recorded by two detectors located at different points of transverse profile. (a) Detector 1; (b) detector 2 ; (c) superposition of the two out-of-phase time traces during a burst of fast oscillations. $L=47 \mathrm{~mm}$.

To study how multimode oscillations are switched on and off when cavity length is varied, we have monitored the evolution of the beam spatial profile as the uncontrolled cavity was slowly drifting due to thermal effects. Sufficient spatial and temporal resolutions could be simultaneously achieved by using a linear CCD array of 256 pixels, with an acquisition rate of 33,000 profiles/second. Fig. 5 shows a typical observation made in the same configuration as in Fig 1 . This allows us to correlate qualitative changes in the total output intensity with modifications in the beam transverse profile.

In Fig. 同, the following events can be seen from left to right. First there is a mode hop, with a sudden jump in the total intensity and a transition from a one-hump transverse profile to a two-hump one. After the mode hop, the output intensity and the two-hump profile remain approximately constant during $200 \mu \mathrm{s}$. Then, the system switches abruptly to finite amplitude oscillations, with no notable change in the transverse profile. As cavity drifts through the oscillation zone, the transverse profile changes gradually from a two-hump profile to a one-hump one until oscillations disappear through an inverse supercritical Hopf bifurcation. Finally, intensity and the one-hump profile remain constant until the end. This scenario indicates that fast oscillations occur between two cavity lengths where two different transverse modes are dominant, as predicted theoretically [11], and that composite profiles are observed in the unstable zone. A singular value decomposition analysis of these profiles confirms that they are linear superpositions of the two monomode profiles.

According to Ref. [11], two-mode regimes should be much easier to observe than if transverse degeneracy were required for mode interaction. In order to estimate the prevalence of fast multimode oscillations in our experimental system, including far from confocal or concentric geometries, we have thus conducted a systematic search near cavity lengths $L=46,63,71,77 \mathrm{~mm}$. Multimode behavior has been easily observed in each case.

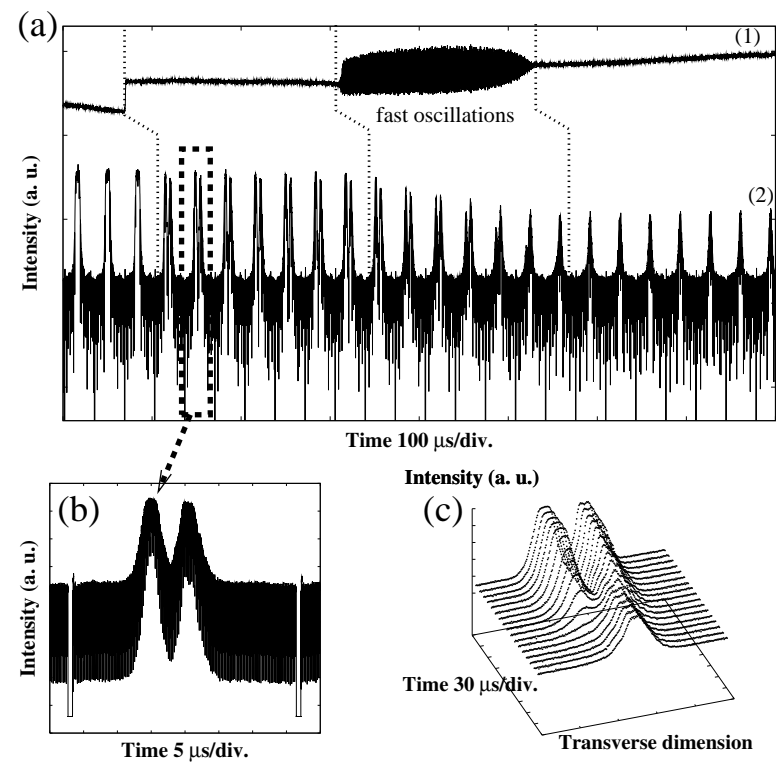

FIG. 5: (a) Traces 1 and 2 show total signal intensity as a function of time and the raw video signal from the CCD array, including interpixel and interframe pulses. Trace 2 is lagging behind trace 1 by the CCD transfer time $(30 \mu \mathrm{s})$, as indicated by dashed lines. (b) The video signal between two interframe pulses displays a one-dimensional section of the beam profile acquired at the beginning of the segment, superimposed with interpixel pulses. (c) Succession of 1D profiles in the oscillation zone, extracted from trace 2 by suitable filtering, showing gradual transition from a two-hump to a single-hump profile.

For example, Fig. 6 displays the result of several cavitylength scans carried out near the configuration of Figs. 3 5, by increments of $200 \mu \mathrm{m}$ or $500 \mu \mathrm{m}$. For each configuration, cavity detuning was swept over one free spectral range to find whether or not fast oscillations could be observed for some detuning. The data of Fig. 6 clearly show that multimode behavior can be observed in almost all configurations studied. Surprisingly, transverse profiles almost always resemble that of low-order modes. Oscillation frequencies gather around $10-15 \mathrm{MHz}$, with a few points in the $100-300 \mathrm{MHz}$ range. For cavity lengths $L=63,71$ and $77 \mathrm{~mm}$, most probable frequencies were in the $6-15 \mathrm{MHz}$, the $3-8 \mathrm{MHz}$ and the $1-3 \mathrm{MHz}$ ranges, respectively, but frequencies well outside these ranges have also been observed. Thus, lower frequencies are seemingly favored as cavity length is increased but we do not yet have an explanation for this behavior.

Note that the degenerate model whose predictions 11, 14. motivated our experiments is not suitable to describe a type-II OPO, where signal and idler fields have orthogonal polarizations. The study of the non-degenerate multimode model is difficult and is currently in progress. As in the degenerate case, cross-conversion channels involve signal and idler fields with different transverse structures, but which now become resonant in symmetrical pairs be- 


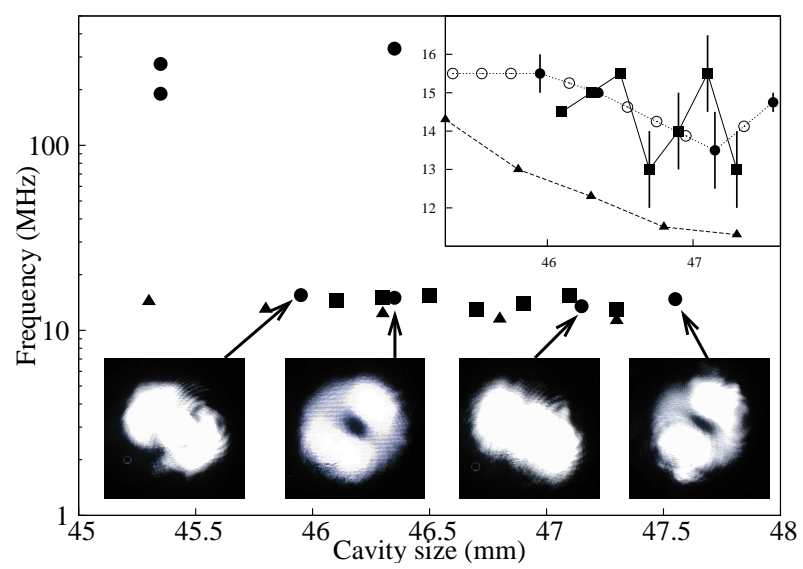

FIG. 6: Frequencies of multimode oscillations observed in three scans (each associated with a different symbol) of cavity length in the $45-48 \mathrm{~mm}$ range. Inset shows data points with frequencies between 10 and $15 \mathrm{MHz}$, with a line passing through data points of a given scan. Filled (open) symbols denote configurations where oscillations were observed (not observed). When different oscillation regimes were observed in the same configuration, the frequency range is indicated by a vertical bar. Transverse profiles observed in the scan associated with circle symbols are also shown.

cause of the small difference between the periods of the signal and idler frequency combs.

In conclusion, we have shown that the fast oscillations observed in TROPOs are generated by the coexistence of interacting transverse modes. The surprising prediction made in Ref. 11] that multimode oscillations do not require mode degeneracy also holds in our system, as confirmed by systematic scans of the cavity lengths which revealed a high probability of occurence. By monitoring the transverse structure of the beam as cavity length was slowly swept, we have also evidenced the occurence of fast oscillations due to mode coexistence between two resonances corresponding to two different transverse modes. These findings are important because they show that two-mode regimes can have a lower threshold than invidual modes and thus must be taken into account to determine which operating mode is selected in OPOs [19, 20.

Finally, this phenomenon should not be specific to OPOs or even optical systems. Similar observations have been reported in hydrodynamical Faraday experiments [7], where the nonlinearity is formally equivalent to the parametric interaction in an OPO. In fact, the simplicity of the basic ingredient, namely that the nonlinear interaction of two non-resonant modes generates a resonant oscillation that sustains multimode behavior, suggests that cooperative multimode oscillations may occur in any system where a nonlinear wave mixing interacts with strong boundary conditions.

We are most grateful to Jaouad Zemmouri for his contribution in the initiation of this research project and for stimulating discussions. CERLA is supported by the Ministère chargé de la Recherche, Région Nord-Pas de Calais and FEDER.

[1] A. E. Siegman, Lasers (University Science Books, 1986).

[2] Y. I. Khanin, Fundamentals of laser dynamics (Cambridge International Science Publishing, 2005).

[3] C. Szwaj, S. Bielawski, and D. Derozier, Phys. Rev. A 57, 3022 (1998).

[4] C. Fabre, P. F. Cohadon, and C. Schwob, Quantum Semiclass. Opt. 9, 165 (1997).

[5] C. Fabre et al., C. R. Acad. Sci. Paris 1 IV, 553 (2000).

[6] P. Manneville, Instabilities, Chaos and Turbulence (Imperial College Press, 2004).

[7] S. Residori, A. Guarino, and U. Bortolozzo, Europhys. Lett. 77, 44003 (2007).

[8] C. Schwob et al., Appl. Phys. B 66, 685 (1998).

[9] M. Vaupel, A. Maitre, and C. Fabre, Phys. Rev. Lett. 83, 5278 (1999).

[10] S. Ducci et al., Phys. Rev. A 64, 023803 (2001).

[11] A. Amon et al., Phys. Rev. A 68, 023801 (2003).

[12] L. A. Lugiato et al., Il Nuovo Cimento 10D, 959 (1988).

[13] C. Richy et al., J. Opt. Soc. Am. B 12, 456 (1995).

[14] P. Suret et al., Opt. Commun. 200, 369 (2001).

[15] P. Suret et al., Phys. Rev. A 61, 021805(R) (2000).

[16] P. Suret et al., Opt. Lett. 26, 1415 (2001).

[17] A. Amon and M. Lefranc, Phys. Rev. Lett. 92, 094101 (2004).

[18] A. Amon and M. Lefranc, Eur. Phys. J. D 44, 547 (2007).

[19] R. C. Eckardt et al., J. Opt. Soc. Am. B 8, 646 (1991).

[20] T. Debuisschert et al., J. Opt. Soc. Am. B 10, 1668 (1993). 